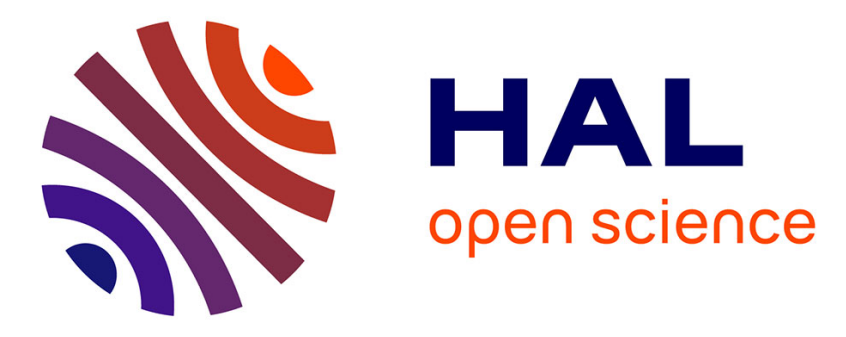

\title{
Abstraction, objet éternel et occurrence actuelle
}

\author{
Frédéric Nef
}

\section{To cite this version:}

Frédéric Nef. Abstraction, objet éternel et occurrence actuelle. François Beets, Michel Dupuis et Michel Weber. La science et le monde moderne d'Alfred North Whitehead Alfred North Whitehead's Science and the Modern World, Ontos Verlag, pp.363-382, 2006, II,5. ijn_00352716

\section{HAL Id: ijn_00352716 https://hal.science/ijn_00352716}

Submitted on 13 Jan 2009

HAL is a multi-disciplinary open access archive for the deposit and dissemination of scientific research documents, whether they are published or not. The documents may come from teaching and research institutions in France or abroad, or from public or private research centers.
L'archive ouverte pluridisciplinaire HAL, est destinée au dépôt et à la diffusion de documents scientifiques de niveau recherche, publiés ou non, émanant des établissements d'enseignement et de recherche français ou étrangers, des laboratoires publics ou privés. 
François Beets, Michel Dupuis et Michel Weber (éditeurs)

\section{La science et le monde moderne d'Alfred North Whitehead}

\section{Alfred North Whitehead's Science and the Modern World}

Actes des Journées d'étude internationales tenues à l'Université catholique de Louvain, les 30-31 mai et 1 juin 2003

Proceedings of the Second International "Chromatiques whiteheadiennes" Conference.

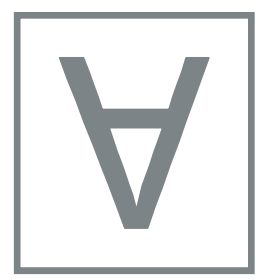

ontos

verlag 
Bibliographic information published by Die Deutsche Bibliothek

Die Deutsche Bibliothek lists this publication in the Deutsche Nationalbibliographie; detailed bibliographic data is available in the Internet at http://dnb.ddb.de

\title{
Publiés avec le concours du FNRS et de la Communauté française de Belgique
}

\author{
North and South America by \\ Transaction Books \\ Rutgers University \\ Piscataway, NJ 08854-8042 \\ trans@transactionpub.com
}

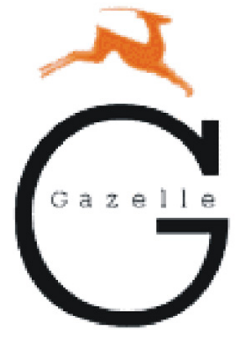

United Kingdom, Ire Iceland, Turkey, Malta, Portugal by

Gazelle Books Services Limited

White Cross Mills

Hightown

LANCASTER, LA1 4XS

sales@gazellebooks.co.uk

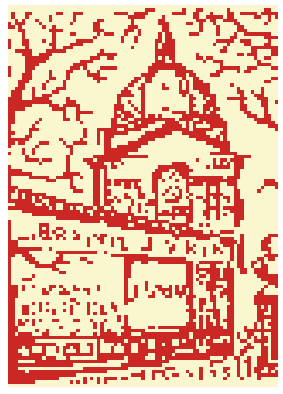

Livraison pour la France et la Belgique:

Librairie Philosophique J.Vrin

6, place de la Sorbonne; F-75005 PARIS

Tel. +33 (0)1 43540347 ; Fax +33 (0)1 43544818

www.vrin.fr

${ }^{\circ} 2006$ ontos verlag

P.O. Box 15 41, D-63133 Heusenstamm

www.ontosverlag.com

ISBN 3-938793-07-4

2006

No part of this book may be reproduced, stored in retrieval systems or transmitted in any form or by any means, electronic, mechanical, photocopying, microfilming, recording or otherwise without written permission from the Publisher, with the exception of any material supplied specifically for the purpose of being entered and executed on a computer system, for exclusive use of the purchaser of the work

Printed on acid-free paper

ISO-Norm 970-6

FSC-certified (Forest Stewardship Council)

This hardcover binding meets the International Library standard

Printed in Germany

by buch bücher $\mathbf{d d}$ ag 


\section{Sommaire - Contents}

Contributors / Liste des auteurs.................................................................................................. 7

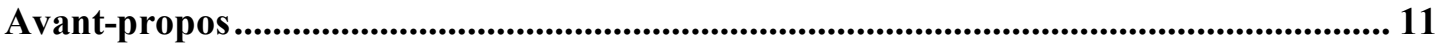

Michel Weber (Louvain-la-Neuve)

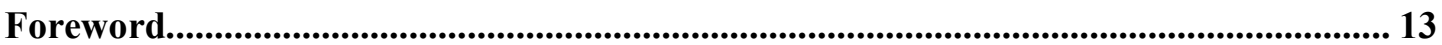

Michel Weber (Louvain-la-Neuve)

Lire Science and the Modern World................................................................................................. 17

François Beets (Liège)

Introduction: A. N. Whitehead Natural Philosopher ............................................................ 23

Jacques Riche (Leuven)

I. La science et le monde moderne - Science and the Modern World....................... 55

1. Faits, ordre des choses et foi scientifique ............................................................................. 57

Xavier Verley (Toulouse le Mirail)

2. Mathematics and Philosophy.................................................................................................. 77

Ivor Grattan-Guinness (Middlesex)

3. Le sophisme du concret mal placé........................................................................................ 81 Guillaume Durand (Nantes)

4. The Organic Turn: From Simple Location to Complex (dis)Location.......................... 97 Michel Weber (Louvain-la-Neuve)

5. The Double Meaning of Whitehead's Notion of Romanticism................................... 119 Luca Vanzago (Pavia)

6. Whitehead and the Rise of Modern Science

Lieven Decock (Amsterdam)

7. Relativité et interconnexité .......................................................................................................... 147

Bertrand Saint-Sernin (Paris)

8. La théorie quantique.

Jean-Michel Counet (Louvain-la-Neuve)

9. L'esprit, l'événement et le temps........................................................................................... 177

Pierre Cassou-Noguès (Lille)

10. Degrés d'abstraction

Ali Benmakhlouf (Nice)

11. No Concretion without God

Palmyre Oomen (Nijmegen)

12. Platonism at Work: Religion, Science, and the Hope of Rationalism

Gottfried Heinemann (Kassel)

13. De l'esthétique à l'histoire. L'atéléologie des valeurs vives 
1. A. N. Whitehead as Precursor of the New Theories of Self-Organization. Alain Beaulieu (McGill)

2. Idéalité et abstraction dans la dernière philosophie de Whitehead 281 Vincent Berne (Paris)

3. Le concept d'abruptness et le principe de translucency of realization Jean-Marie Breuvart (Lille)

4. Poésie, ontologie et mathématiques Jean-Claude Dumoncel (Caen)

5. Abstraction, objet éternel et occurrence actuelle 363 Frédéric Nef (EHESS)

6. Merleau-Ponty, lecteur de Whitehead Franck Robert (Barcelonnette)

7. Deleuze and Whitehead on the Concept of Reciprocal Determination. James Williams (Dundee)

Bibliographies

Table des matières - Table of Contents

Collection « chromatiques whiteheadienne » 


\section{Contributors / Liste des auteurs}

Alain Beaulieu completed a Ph.D. in Philosophy at the University of Paris VIII. He teaches philosophy at McGill University and at the Université du Québec à Montréal (Canada). He is the editor of Gilles Deleuze. Héritage philosophique (PUF, 2005) and the coeditor (with D. Gabbard) of Michel Foucault and Power Today. International Multidisciplinary Studies in the History of Our Present (Lexington Books, 2005). He has also published Gilles Deleuze et la phénoménologie (Sils Maria/Vrin, 2004).

François Beets est professeur à l'université de Liège, où il enseigne la logique, l'épistémologie et la philosophie médiévale. Il a récemment coédité Alfred North Whitehead. De l'algèbre universelle à la théologie naturelle (ontos verlag, 2004) et Logique et ontologie: Perspectives diachronique et synchronique (Université de Liège, 2005).

Ali Benmakhlouf est professeur à l'université Nice-Sophia Antipolis. Spécialiste de logique, il a publié des ouvrages sur Averroès, Frege et Russell et a coordonné le collectif Alfred North Whitehead, l'Univers solidaire (Université de Paris X - Nanterre, 1999).

Jean-Marie Breuvart est professeur retraité de philosophie de l'université catholique de Lille, où il était Directeur du Département d'Éducation. Il a présenté en 1976 à l'université Charles de Gaulle (Lille), une thèse analysant les modèles qui régissent la Philosophie de Whitehead de la symbolisation. Ses publications sont, entre autres, les suivantes: un chapitre dans le livre d'I. Stengers L'Effet Whitehead (Vrin 1994); un chapitre dans le livre Familles et Cultures (ANPASE, 1998); la coordination du livre Migrations, Interculturalité et Démocratie, Septentrion, 1998 ; un chapitre dans le livre de J.F. Rey Spiritualités du temps présent (L'Harmattan, 1999); un chapitre dans le livre d'Alain Jenny La part du Tiers (Romillat, 2001) ; la coordination du livre Les Rythmes Educatifs dans la Philosophie de Whitehead (ontos verlag, 2005).

Vincent Berne est professeur de philosophie dans le XVe arrondissement à Paris. Il est titulaire d'un DEA de l'Université de Paris I (avec un mémoire consacré au problème de l'individuation dans la dernière philosophie de Whitehead) et poursuit ses recherches sur la métaphysique du procès dans le cadre du réseau chromatiques. 
Pierre Cassou-Noguès est chargé de recherches au CNRS, UMR Savoirs, textes languages. Il a notamment publié De l'expérience mathématique (Vrin, 2001) et Gödel (Les belles lettres, 2004).

Dr. Jean-Michel Counet is Professor of medieval philosophy and theology at the University of Louvain-la-Neuve. His main publications are "Ontologie et itinéraire spirituel chez maître Eckhart» (Revue Philosophique de Louvain 96, 1998, pp. 254-280); Mathématiques et dialectique chez Nicolas de Cuse (Vrin, 2000); "Le temps comme explication de l'éternité chez Nicolas de Cues » (Revue Philosophique de Louvain 101, 2003, pp. 319-339); " The meaning of apology and reconciliation for an apophatic theology " (in Conflict and Reconciliation: Perspectives on Nicholas of Cusa, ed. I. Bocken, Brill, 2004, pp. 195-204); "Mathematics and the Divine in Nicholas of Cusa " (in Mathematics and the Divine, ed. T. Koetsier et L. Bergmans, Elsevier, 2005, pp. 273-290).

Dr. Lieven Decock is assistant Professor at the Faculty of Philosophy, Free University of Amsterstam, The Netherlands. He has published Trading Ontology for Ideology. The Interplay of Logic, Set Theory and Semantics in Quine's Philosophy (Dordrecht, 2002).

Jean-Claude Dumoncel enseigne la logique de la théologie au Centre d'Etudes Théologiques de Caen et participe au groupe Academos des Archives Poincaré de Nancy. Il a publié entre autres Le Jeu de Wittgenstein (PUF, 1991), Les 7 Mots de Whitehead (L'Unebévue, 1998), La Philosophie Telle-Quelle (Pétra, 2004) et travaille actuellement à un Tractatus Theologico-Philosophicus.

Guillaume Durand, Docteur en Philosophie avec une thèse sur La méthode de l'abstraction extensive: des événements aux objets dans la philosophie de la nature d'A. N. Whitehead, est Chargé de cours à l'Université de Nantes.

Ivor Grattan-Guinness, holder of both the doctorate (Ph.D.) and higher doctorate (D.Sc.) in the history of science at the University of London, is Emeritus Professor of the History of Mathematics and Logic at Middlesex University, England. His individual books include Convolutions in French mathematics, 1800-1840, three volumes (1990, Birkhäuser); The Fontana and Norton history of the mathematical sciences. The rainbow of mathematics (1997, Fontana; 1998, Norton) and The search for mathematical roots, 1870-1940. Logics, set theories and the foundations of mathematics from Cantor through Russell to Gödel (2000, Princeton University Press). He edited a large collection of essays on Landmark writings in Western mathematics, 1640-1940 (2005, Amsterdam, Elsevier). 
Dr. Gottfried Heinemann is Professor of philosophy at the University of Kassel (Germany). His publications include: Zeitbegriffe (ed.), FreiburgMünchen 1986; Natura desiderata. Das Abstrakte am 'Gebrauchswert' und die Antinomien der Mathematik, Freiburg-München 1988 ; Technê und Physis. Drei Vorlesungen zum griechischen Naturbegriff, Kassel 1999 ; Studien zum griechischen Naturbegriff, Teil I: Philosophische Grundlegung: Der Naturbegriff und die "Natur", Trier 2001.

Frédéric Nef, né en 1947, antérieurement chercheur au CNRS (19801987) et professeur de philosophie du langage à l'université de Rennes I (1987-2003), actuellement directeur d'études à l'EHESS, membre de l'Institut Jean-Nicod, (CNRS/ENS Paris), associé au Groupe de Sociologie Morale et Politique (CNRS), a écrit entre autres : L'Objet Quelconque (Vrin, 1998), Qu'est-ce que la métaphysique? (Gallimard, 2004), Les propriétés des Choses (Vrin, 2006). Ses thèmes de recherche sont d'une part l'ontologie du social et d'autres part la métaphysique générale.

Dr. Palmyre Oomen is Radboud Professor of philosophy at the Eindhoven University of Technology, and chairs the section on theology and science of the Heyendaal Instituut at the Radboud University Nijmegen, both in the Netherlands. Her publications include: Doet God ertoe? Een interpretatie van Whitehead als bijdrage aan een theologie van Gods handelen, Kampen 1998 (2nd revised edition 2004)

Jacques Riche studied philosophy and linguistics at the University of Louvain and received his Ph.D. in logic from the Australian National University. He works on logic and artificial intelligence at the University of Leuven.

Franck Robert, agrégé et docteur en philosophie, enseigne la philosophie au lycée de Barcelonnette. Ses travaux portent sur la philosophie de Merleau-Ponty et le mouvement phénoménologique, sur l'œuvre de Proust et l'ontologie de Whitehead. Il a publié récemment Phénoménologie et Ontologie. Merleau-Ponty lecteur de Husserl et Heidegger, chez L'Harmattan.

Pierre Rodrigo, agrégé de l'Université et docteur en philosophie, est actuellement Professeur à l'Université de Bourgogne (Dijon). Ses recherches portent sur la philosophie aristotélicienne, sur l'esthétique et sur la phénoménologie. Il a publié sur ces questions : Aristote, l'eidétique et la phénoménologie (Millon, 1995) ; Aristote ou l'unité du multiple (Ellipses, 1997) ; Aristote et les 'choses humaines', (Ousia, 1998); L'Étoffe de l'art (Desclée de Brouwer, 2001); "Merleau-Ponty et la psychanalyse», Chiasmi International, 4, 2002; "L'épreuve du sentir. Fondation et refondations de la phénoménologie transcendantale », ALTER, 11, 2003. 
Dans ses travaux l'accent est mis sur la pensée du sensible et de l'être-endevenir. L'aristotélisme et la phénoménologie constituent, dans cette perspective, les deux appuis essentiels de ses recherches, mais il est aussi attentif aux apports de la Process philosophy d'A. N. Whitehead (coordination du $\mathrm{n}^{\circ}$ spécial «Whitehead» des Études Philosophiques, 2002-4) et de la pensée du devenir chez Bergson (Introduction et Commentaire de La Pensée et le mouvant, Ellipses, 1998).

Bertrand Saint-Sernin est professeur émérite à l'université de ParisSorbonne (Paris IV), où il enseignait la philosophie des sciences. Il a été recteur d'Académie. Ses travaux portent sur la philosophie de l'action (les mathématiques de la décison), d'une part, sur la philosophie de la nature, de l'autre. Il a engagé une enquête sur l'état de la raison aujourd'hui.

Luca Vanzago graduated at Pavia University, Italy, with a dissertation devoted to Whitehead's concept of time. His subsequent research developed along two main lines: the ontology of processes and events and its categorical framework, including logical and epistemological issues; the problem of manifestation and relations. This trend of research constitutes the ground of his $\mathrm{PhD}$ dissertation (Katholieke Universiteit Leuven) devoted to a comparison between Whitehead's and Merleau-Ponty's theories, which was published in Italian. More recently Vanzago has published a monograph devoted to Whitehead's notion of process, as well as one concerning Husserl's conception of consciousness, and is working at a monograph devoted to the phenomenology of the embodied experience. Luca Vanzago works at the Department of Philosophy of Pavia University, Italy.

Dr. Xavier Verley is Professeur agrégé and senior lecturer at the Université de Toulouse le Mirail. His research interests are in symbolic logic, epistemology, philosophy of sciences and psychology. He has published on Mach, Carnap, Frege, Husserl and Whitehead.

Dr. James Williams teaches philosophy at the University of Dundee. His publications include Lyotard: Towards a Postmodern Philosophy (Cambridge: Polity), Lyotard and the Political (Routledge) and Gilles Deleuze's Difference and Repetition: a Critical Introduction and Guide (Edinburgh University Press). 


\section{Abstraction, objet éternel et occurrence actuelle}

\section{Frédéric Nef (EHESS, Institut Jean-Nicod et Institut Marcel-Mauss)}

Il s'agira ici d'évaluer la cohérence de la doctrine des objets éternels à la lumière des développements récents de la métaphysique des relations internes. On sait que la théorie de l'abstraction de Whitehead a été soumise à une critique très sévère de $J$. Vuillemin $(1971)^{1}$, qui n'a cependant pas pris en compte ce texte très difficile. Il serait donc sans doute opportun d'ouvrir à nouveau ce dossier, afin de déterminer la valeur des aperçus du maître de Harvard.

Le chapitre "Abstraction» (ainsi que celui sur Dieu, dont il est inséparable ${ }^{2}$ ) a été ajouté après coup aux Lowell Lectures qui ont été prononcées en février 1925 à Cambridge, Massachussets. Les Lowell Lectures concernent la philosophie de la science naturelle. Whitehead affirme dès 1919 que l'essence de la nature est le passage. Dans les Lowell Lectures the creative advance of nature est conçue comme un résultat d'une underlying activity of realization. La question posée dans SMW porte sur ce qui émerge dans l'actualité des événements. La réponse est : la valeur - value is the word I use for the intrinsic reality of an event, déclare par exemple Whitehead, qui introduit à ce propos le concept d'éternalité (eternality) distingué de celui de d'éternité. L'eternality est un realm of eternal objects, comme les formes, couleurs, contours, caractères... qu'un événement peut manifester. C'est dans le chapitre que je me propose d'examiner que ce deuxième aspect de l'événement (à côté de la valeur) est mis en lumière.

Si SMW expose une philosophie de la nature qui doit intégrer les données de la physique contemporaine, la perspective de ce livre n'est pas seulement épistémologique, mais aussi métaphysique - comme il le rappelle opportunément dans la préface, il entend proposer une alternative au matérialisme scientifique, exactement comme Leibniz à Hobbes ou Aristote aux atomistes. C'est dans cette perspective qu'il faut situer son ontologie des propriétés émergentes, dont la source est Llyod Morgan et Samuel Alexander, beaucoup plus que Bergson, cité à deux reprises. V . Lowe (II, p. 173) rapporte que Whitehead, âgé, lui avait déclaré qu'en 1925, date des Lowell lectures, S. Alexander était le philosophe qui lui importait le plus $^{3}$ et qu'il le considérait toujours comme une synthèse 
d'universel et d'individuel, une combinaison de Spinoza et de Leibniz, synthèse qu'il visait pour son propre compte. Pour Alexander, comme pour Whitehead, l'expérience fondamentale est celle de la comprésence ${ }^{4}$ ou de la togetherness, et non celle des sense data: il y a préhension parce qu'il y a comprésence et non parce qu'il y a des sense data.

Je commencerai par commenter le chapitre «Abstraction" de manière relativement détaillée, après avoir rappelé quelques éléments de la théorie des objets éternels, fondamentaux et pour la pensée de Whitehead en général et pour ce texte en particulier. Je me livrerai ensuite à une évaluation succincte de la cohérence de cette théorie, notamment en ce qui concerne les relations internes. ${ }^{5}$

\section{Les objets éternels}

L'ontologie de Whitehead est une ontologie d'événements, qui ont pour caractéristique d'apparaître et de disparaître. Pour connaître quelque chose, il faut une stabilité, un objet, que Whitehead considère non comme des qualités des événements (leur stabilité, leur objectivité qui les rendrait connaissables), mais comme ce qui permet l'ingression ${ }^{6}$ d'un objet dans l'événement. L'ingression d'après The Concept of Nature est « la manière dont le caractère de l'événement se façonnera en raison d'être de l'objet. C'est-à-dire que l'événement est ce qu'il est, parce que l'objet est ce qu'il est [...; il est également vrai de dire que les objets sont ce qu'ils sont parce qu'ils sont ce qu'ils sont ${ }^{7} »$.

Pour Whitehead il y a un nombre indéfini de types d'objets, mais on peut se limiter à trois principaux: objets des sens (sense objects) ${ }^{8}$, objets perceptuels, objets scientifiques. La différence entre les deux premiers est proche de celle qui existe entre sensation et perception, mais ne lui est pas identique. Les objets des sens sont les couleurs, les sons, les odeurs; les objets perceptuels sont des associations d'objets des sens - ils sont des objets que l'on appelle les 'objets physiques' (chaises, tables...). Les objets scientifiques sont des caractères ou aspects les plus fondamentaux des événements (exemple : électron). L'objet scientifique est toujours solidaire d'un champ théorique'.

Les objets éternels sont introduits quand Whitehead passe d'un point de vue épistémologique à une perspective ontologique ${ }^{10}$. Ils sont introduits pour répondre à cette question: d'où vient à l'entité actuelle sa forme déterminée ? L'expression « objet éternel » vient de ce que d'une part, à la différence des événements, ils sont éternels pour être connus et que d'autre part Whitehead se refuse à utiliser l'expression « universaux », qui écrase 
toute la dialectique de l'individuel et de l'universel dans l'objet. Les objets éternels sont des entités non actuelles en elles-mêmes, mais exemplifiées selon une relation de pertinence (relevance) dans tout ce qui est actuel. Les objets actuels sont les «purs potentiels de l'univers et les entités actuelles diffèrent les unes des autres dans leur réalisation des potentiels » (PR, p. 226). Ce sont des «purs potentiels pour la détermination du fait» (PR, p. $32)$.

L'objet éternel est abstrait de toute entité actuelle particulière. C'est une potentialité en vue d'une ingression. Il possède ainsi une nature modale (on y reviendra) ; c'est un possible pour une actualité. Il est transcendant car il ne dépend d'aucune occasion actuelle, tout en étant non séparé, à la différence des formes platoniciennes. Ce n'est pas pour autant un universel aristotélicien ${ }^{11}$. Whitehead pense que ce dernier dépend trop étroitement de la différence species/genera, trop limité donc à un schème conceptuel classificatoire trop étroit pour une véritable philosophie de l'organisme ${ }^{12}$. Un objet éternel est une essence individuelle ; c'est donc un particulier. Son essence relationnelle consiste dans sa relation aux autres objets éternels ${ }^{13}$.

Les objets éternels sont donc des abstraits particuliers, transcendants et relationnels. Ils sont différents à la fois des objets de la perception et des objets scientifiques. Ce sont des entités qui sont du type ni des chaises, ni des électrons, ni à plus forte raison des odeurs ou des sons.

\section{Abstraction, relation et particularité}

Le chapitre «Abstraction» des Lowell Lectures remplit au moins trois objectifs : montrer la nature de la relation entre les objets éternels et les occasions actuelles, développer le lien entre essence individuelle et essence relationnelle et enfin exposer le rapport entre potentialité générale et potentialité réelle dans le continuum spatio-temporel ${ }^{14}$. Le premier objectif touche avec la question des relations internes : la relation de l'objet éternel à l'objet actuel est externe, alors que la relation de l'objet externe à l'objet actuel est interne. On reviendra sur ce point central, mais il faut déjà mentionner, pour ne pas rendre cette affirmation par trop énigmatique, que la relation est interne en ceci que l'objet actuel a nécessairement une relation à l'objet éternel concerné, alors qu'elle est externe en ceci que l'objet éternel n'est pas nécessairement exemplifié dans cet objet actuel. Notons que la relation entre $\mathrm{A}$ et $\mathrm{B}$ n'a pas forcément le même caractère que la relation entre $\mathrm{B}$ et $\mathrm{A}$.

Whitehead commence par distinguer occasion of cognition et occasion of experience, ce qui correspond en gros à la distinction que nous faisons 
actuellement entre le contenu conceptuel et le contenu non conceptuel. Il prend comme exemple a definite shade of red et the shape of sphericity ${ }^{15}$ qui peuvent s'impliquer dans l'occasion actuelle mais qui la transcendent, car elles peuvent toutes deux avoir d'autres relations à d'autres occasions. Le premier des deux exemples a ceci d'intéressant qu'il est un particulier (alors que le second est un universel). Whitehead analyse la constitution ontologique d'un particulier concret, par exemple une balle d'une nuance particulière de rouge.

Une occasion actuelle est définie ainsi : «a set within a realm of alternative interconnected entities ». Jusqu'ici on ne dépasse pas la perception d'un espace logique de prédication: Red (a) \& spherical (a), mais aussi Blue (b) \& spherical (b) \& Red (a) \& Non spherical (a)... Cet espace logique de prédication est celui d'un domaine de suggestions alternatives.

La thèse centrale de Whitehead est que l'analyse métaphysique de l'actualité exige une référence à l'idéalité ${ }^{16}$ : Dans la "situation métaphysique », les deux domaines d'actualité et d'idéalité sont inséparables (intrinsically inherent). Les objets actuels sont composés d'objets idéaux, les particuliers concrets sont des totalités dynamiques de particuliers abstraits. Whitehead appelle les objets éternels des entités transcendantales, idéales et insiste sur leur aspect abstrait. Ce terme a deux sens : d'une part $x$ est abstrait si $x$ peut être compris isolément ${ }^{17}$, d'autre part $\mathrm{x}$ est abstrait si $\mathrm{x}$ transcende toute occasion concrète $\mathrm{y}$, c'est-à-dire si $\mathrm{x}$ ne dépend pas de $\mathrm{y}, \mathrm{y}$ étant n'importe quelle occasion concrète ${ }^{18}$. L'abstraction est compatible avec la connexion ${ }^{19}$. Cette connexion c'est l'ingression dans une occasion :

Si $\mathrm{x}$ a une relation d'ingression dans $\mathrm{y}$, $\mathrm{x}$ est connecté à $\mathrm{y}$, même si $x$ ne dépend pas de $y$. Si $x$ a une relation d'ingression dans $y$, on dira alors que $\mathrm{x}$ est connecté à $\mathrm{y}$ par une relation de connexion indépendante.

Cette relation de connexion indépendante est différente de la relation tout/partie dans la méréologie de Whitehead : habituellement une partie $\mathrm{x}$ est connectée à un tout $\mathrm{y}$, si $\mathrm{x}$ dépend de $\mathrm{y}$ (par exemple un doigt et la main à laquelle il appartient).

Le premier principe métaphysique que Whitehead dégage à propos des objets éternels est leur individualité. Il y a une individualité de l'universel et ceci ne doit pas être compris au sens d'une identité des contraires, comme dans la dialectique hégélienne, mais plutôt au sens d'une coincidentia oppositorum (Nicolas de Cues ${ }^{20}$ ). Whitehead va quelquefois 
jusqu'à appeler un objet éternel un particulier, ce qui pose évidemment un problème logique, mais il entend par là qu'il y a une distinction numérique entre les objets éternels. Par exemple la sphéricité de la Terre est distincte numériquement de celle de Mars, même en supposant que ces deux planètes sont idéalement sphériques et que donc leurs sphéricités soient indiscernables (cf. supra). Il ne faut pas confondre deux ordres de distinction et Whitehead n'est pas complètement soigneux à ce propos : un objet éternel est distinct d'un autre objet éternel par le type de généricité (la sphéricité de la balle est distincte de sa couleur) et de plus un objet éternel est distinct d'un autre objet éternel par son ingression : la sphéricité de cette balle est distincte de la sphéricité de cette autre balle. Cette individualité de l'objet éternel est ce que l'on nomme traditionnellement, pour Whitehead, essence individuelle, ou plus exactement cette dernière est constituée $\mathrm{d}^{\prime}$ 'objets éternels ${ }^{21}$. Dans la mesure où un objet éternel possède une relation d'ingression avec un objet actuel $b$, a appartient à l'essence individuelle de b. Donc, d'une part un objet éternel est ce que l'on pourrait appeler l'haeccéité comme essence individuelle et, d'autre part, l'objet éternel est ce qui peut être considéré comme donnant à une occurrence actuelle sa concrétion. La balle en question est concrète, dans la mesure où elle est sphérique, rouge etc. et elle est ce qu'elle est. L'individualité de l'objet éternel est liée à la particularité de l'occurrence actuelle ${ }^{22}$ C'est sûrement en ce sens que l'on peut peut-être parler avec Whitehead de la particularité de l'objet éternel, héritée en fait de celle de l'occurrence actuelle. En termes un peu scolastiques, on peut dire que l'individualité de l'objet éternel (expression moins dangereuse) est composée de l'individualité de l'acte d'ingression et de l'individualité de l'essence. Cette caractérisation par l'essence individuelle est formelle; il faut préciser le statut métaphysique de l'objet éternel et on se souvient que Whitehead le caractérise comme une possibilité en vue d'une actualité. Si aucune occurrence actuelle n'était en relation d'ingression avec cette nuance de rouge, celle-ci serait une possibilité, en vue d'une actualité, par l'entremise d'une ingression toujours possible. Cette essence individuelle possible en vue de l'actualité caractérise l'objet éternel et en cela il est fort différent de la Forme platonicienne qui n'est pas un être en vue de l'actuel, mais une actualité pure, participée ou pas.

Le deuxième principe métaphysique fondamental est que l'occurrence actuelle, comme entité abstraite, ne peut être séparée de la référence à d'autres objets éternels et de sa référence à l'actualité. Cela signifie qu'à côté, ou plutôt de manière inséparable de l'essence individuelle, il y a une essence relationnelle de l'occurrence actuelle. Cette essence relationnelle est composée d'une relation déterminée à d'autres objets éternels et d'une relation indéterminée à des occurrences actuelles. C'est ici que nous 
rencontrons la thématique des relations internes: la relation de l'objet éternel à d'autres objets éternels est déterminée et découle de l'essence de l'objet éternel de manière déterminée; c'est donc une relation interne. Whitehead définit ainsi ce type de relation : A a avec B une relation interne $R$ si la relation $R$ est déterminée et si $R$ est constitutive de $A^{23}$. Whitehead ajoute un autre élément définitionnel : une relation $\mathrm{R}$ est interne si elle est éternelle, c'est-à-dire si $\mathrm{R}$ lie $\mathrm{A}$ et $\mathrm{B}$ à tout moment du temps (postérieur à l'existence de A et de B).

Whitehead n'attribue pas la même propriété à la relation et à sa converse : la relation de l'indétermination de l'objet éternel $(\mathrm{A})$ à la détermination de l'occasion actuelle $(\alpha)$ est interne; celle de $(\alpha)$ à $(\mathrm{A})$ est externe ${ }^{24}$. On reviendra sur ce point difficile, lorsque nous aurons à évaluer la cohérence de cet ensemble d'idées. On pourrait redouter au premier abord qu'il ne soit d'emblée incohérent d'attribuer à une même relation la propriété d'être interne et externe. Cependant on peut remarquer que $(\alpha)$ est la converse de (A) : il s'agit donc plutôt d'attribuer à une relation et à sa converse une propriété différente d'internalité. Nous reconnaissons souvent une telle asymétrie, par exemple à propos des actes psychiques. Si je me remémore un événement en entendant une conversation, il existe une relation interne entre la remémoration et l'audition (il y a une relation nécessaire entre la première et la seconde, car la remémoration est fondée sur l'audition) et externe entre l'audition et la remémoration ( $j$ 'aurais pu me remémorer cet événement à une autre occasion). Whitehead affirme de même que la relation d'une nuance de bleu à une occasion actuelle qui l'exemplifie est interne, alors que la relation de cette occasion actuelle à cette nuance de bleu exemplifiée est externe ${ }^{25}$. Il faut donc distinguer ce que l'on nomme habituellement exemplification de A dans $\alpha$ (ingression de A dans $\alpha$ ) de la prédication de $\mathrm{A}$ au sujet de $\alpha$. L'exemplification est déterminée et constitutive, tandis que la prédication est relativement indéterminée et non constitutive. On peut reformuler dans un cadre aristotélicien ce lien entre les deux

$\exists \mathrm{R} \exists \mathrm{S}[(\mathrm{R}: \alpha$ est l'actualisation de $\mathrm{A}) \Leftrightarrow(\mathrm{S}: \mathrm{A}$ est attribué à $\left.\alpha)^{26} \& \operatorname{Cnv}(\mathrm{R}, \mathrm{S})\right]^{27}$

où $\mathrm{R}$ est interne et $\mathrm{S}$ externe. On peut penser ces deux relations sous le chef plus général de la connexion et c'est en pensant à la connexion entre $\mathrm{A}$ et $\alpha$ que Whitehead conçoit une relation qui dans ce cas d'après son orientation est interne ou externe. La connexion interne est évidemment plus forte que la connexion externe, ce qui est une variante de la thèse de la survenance de la vérité sur l'être, du sémantique sur l'ontologique ${ }^{28}$. 
$\alpha$ est définie comme préhension synthétique, ce qui est une manière de caractériser la fonction métaphysique de l'occasion actuelle. Celle-ci sera qualifiée de synthèse esthétique de l'occasion actuelle et des essences individuelles de l'objet éternel, synthèse esthétique car elle apparaît de manière sensible. Ceci implique que pour Whitehead la togetherness puisse être l'objet d'une préhension sensible. Peut-elle être perçue ? Quelle forme prend cette préhension synthétique ${ }^{29}$ ? La préhension synthétique est la solution de l'indéterminité de $\mathrm{A}$ dans la déterminité de $\alpha$ : la synthèse est celle de la déterminité et de l'indéterminité, car, comme on le verra, $\alpha$ est être et non être. C'est la relationalité déterminée de A qui représente une possibilité pour la réalisation - si A restait indéterminé, son être en vue de l'actualité ne se réaliserait pas - c'est le fait que A soit relationnel qui permet à cet être en vue de l'actualité de se réaliser dans une préhension synthétique, une synthèse esthétique. A cette occasion Whitehead énonce ce qui pourrait bien être un axiome de sa théorie de l'abstraction :

(Axiome 1). Il y a un fait général de relationalité mutuelle et systématique qui est inhérent au caractère de la possibilité.

La nature même de la relation explique cet axiome: Une relation qui connecte des relata ne peut porter sur un seul de ses relata. Une possibilité est relationnelle, d'une part parce qu'elle est en vue de l'actualisation, d'autre part parce qu'elle est toujours en relation avec d'autre possibilité ${ }^{30}$. Les possibles ne sont pas mis en relation de manière extrinsèque ou post hoc les uns avec les autres, ou avec des occasions actuelles; leur relationalité est intrinsèque. Partant, leur connexion est nécessaire et non pas accidentelle. La connexion est nécessaire entre les objets éternels est nécessaire, contingente entre les occasions actuelles et les objets éternels, au sens où la plupart des propriétés des occasions actuelles ne sont pas nécessaires. Cependant, si un objet éternel a une ingression dans une occasion actuelle, alors c'est une nécessité pour l'objet éternel de l'avoir. On retrouve dans cette asymétrie entre nécessité et contingence l'équivalent modal de l'asymétrie entre interne et externe, ce qui est normal dans la mesure où ce qui est interne est nécessaire et ce qui est externe est en général contingent.

Ce qui manque chez un métaphysicien certes spéculatif, mais systématique comme Whitehead c'est une axiomatique. On voit très bien les raisons qu'il a de ne pas axiomatiser son ontologie. Quand il a essayé de dresser une liste de ses principes métaphysiques, au début de Process and reality, le résultat n'a pas été convaincant, car il y a trop de principes et certaines notions fondamentales ne sont pas définies et donc restent très obscures, ce qui a détourné de sa philosophie les métaphysiciens épris de 
clarté (à l'exception notable de Peter Simons). Cependant il peut être utile au commentateur et au philosophe de s'imposer une explicitation des axiomes fondamentaux de cette théorie ne serait ce que pour permettre à la contestation de l'interprétation ou de l'évaluation qu'il propose de prendre une forme précise. Le second axiome de la théorie de l'abstraction complète le premier et le troisième est nécessaire pour articuler ce qu'il en est des relations internes :

(Axiome 2). Toute relation qui est possible est dans le domaine de la possibilité.

Cet axiome est très puissant, car il énonce qu'il n'y a pas de relation isolée des autres relations (l'axiome 1 assurant qu'il n'y a pas de possibilité sans relation). En effet toute relation $\mathrm{R}$ possible sera dans le domaine $\mathrm{D}$ des relations possibles. $\mathrm{Y}$ aurait-il donc une métarelation $\mathrm{S}$ dans $\mathrm{D}$ de $\mathrm{R}$ avec toutes les relations possibles ? On pourrait redouter dans ce cas que cela n'entraîne une régression infinie des relations "à la Bradley »" : n'y aurait-il pas alors un domaine D' des métarelations du second ordre et donc une métarelation S' de troisième ordre etc. ? Il n'en est rien car nous savons par l'axiome 1 qu'il y a une relationalité intrinsèque à la possibilité. Cela signifie que plongée dans $\mathrm{D}, \mathrm{R}$ est intrinsèquement en relation avec les autres relations possibles, sans qu'il soit besoin d'une métarelation ou de relations supplémentaires.

(Axiome 3). Tout objet éternel se tient de manière interne dans toutes ses relations possible. ${ }^{32}$

Cela ne signifie pas que toutes les qualités d'un particulier sont nécessaires, ce qui est manifestement faux à moins d'épouser une forme de superessentialisme. ${ }^{33}$ Whitehead affirme, ce qui est très différent, que tout objet éternel a un ensemble de relations possibles et qu'il a ces relations possibles de manière externe. On savait que dans la synthèse préhensive la relation de $\mathrm{A}$ à $\alpha$ était interne. L'axiome 3 dit que toutes les relations possibles de A aux occasion actuelles $\beta, \gamma, \delta$ sont internes.

Il faut distinguer les relations entre objets éternels et entre ceux-ci et les occasions actuelles : il y a une togetherness ${ }^{34}$ des objets éternels dans les occasions actuelles et leur être ensemble provient de leur relationalité - ils ne sont pas mis ensembles dans les occasions actuelles comme dans des boîtes, mais ils sont en relations les uns avec les autres déjà comme des possibles en vue de l'actualisation et actualisés leurs relations forment la trame qualitative des actualités. Si Whitehead n'utilise pas le terme « comprésence » (que Russell utilisera), c'est sans doute pour éviter les 
connotations temporelles de ce terme. En effet si la relation était « être dans la même occasion actuelle au même moment ", cela ouvrirait la porte à une difficulté supplémentaire: des objets éternels pourraient être des ingrédients d'une occasion actuelle à un certain moment et pas à un autre et dans ce cas il serait compliqué de déterminer la structure temporelle de la comprésence, pour des raisons évidentes. La togetherness étant en fin de compte d'essence modale, la structure temporelle découle d'une structure modale ce qui semble plus satisfaisant. Dans le cas d'une déformation cyclique d'un solide, sous la pression d'un choc répété, l'objet éternel « élasticité » qui est dans l'occasion actuelle du solide n'aura pas, tel un ludion, à apparaître et disparaître. La togetherness est une notion très abstraite, beaucoup plus générale que la notion de co-localité, dans la mesure où il ne faut définir qu'une relation de voisinage maximal et pas l'appartenance à un même voisinage.

Cette togetherness réalisée a comme achèvement une valeur émergente, définie comme relationalité définie et éternelle relativement à laquelle la togetherness actuelle ou réelle est achevée. Ceci conduit à une ontologie extrêmement comprimée où la relationalité éternelle est la forme, l'occasion, le superjet de la valeur informée. Pour Whitehead il y a une fusion de l'essence individuelle et des objets éternels dans une occasion individuelle émergente. Ceci provient d'une complexe hiérarchie abstractive des objets éternels, mais on peut le comprendre même à partir simplement de la relationalité des objets éternels ou possibilités. Une occasion individuelle émergente est donc la fusion de l'essence individuelle d'un objet éternel et des autres objets éternels. Ceci a une conséquence positive : on explique très bien que si la hiérarchie abstractive relative à une occasion actuelle est infinie, on ne peut avoir une description complète d'une occasion actuelle et on explique beaucoup mieux que dans la phénoménologie l'épaisseur, la chair des objets, qui n'est rien de plus qu'un vague épistémique ${ }^{35}$. Il y a cependant une conséquence négative dérivée : toute occasion actuelle est infinie. D'autre part comme il est constitué de part en part d'objets éternels, il est éternel. Ceci doit être associé à la doctrine suivant laquelle l'objet actuel est un mixte de non être et d'être, le non être étant entendu comme une possibilité, et non comme une privation.

Il reste un dernier principe métaphysique fondamental, la «translucidité ${ }^{36}$ de la réalisation » (translucency ${ }^{37}$ of realization). L'impossibilité de la description complète pourrait conduire à une sorte d'ineffabilité du concret sensible, que Whitehead donne les moyens d'éviter. Le choix du terme translucency est très réfléchi, car Whitehead ne veut pas affirmer qu'on a accès, par la perception ou l'analyse métaphysique, à la totalité 
parfaitement articulée de la structure ontologique des occasions actuelles et celles-ci laissent suffisamment de lumière, mais ne représentent en rien un medium neutre pour la perception de leur structure. La translucency est intermédiaire entre la pure diaphanéité et l'opacité ; on peut l'attribuer par exemple à des cristaux qui laissent passer les photons, mais déforment les images, ou à une vitre sale qui laisse deviner grossièrement le paysage ${ }^{38}$. Nous sommes conduits au quatrième axiome métaphysique :

(Axiome 4.) Chaque objet éternel est lui-même, quel que soit le mode de réalisation dans lequel il est présent (c'est-à-dire avec lequel il entre dans une relation d'ingression).

Cet axiome écarte à la fois l'illusion transcendantale et le mobilisme héraclitéen ; c'est le fondement de la vérité-correspondance. La multiplicité des modes d'ingression est une garantie métaphysique pour la survenance de la sémantique sur l'ontologie.

\section{Evaluation critique de la théorie de l'abstraction}

Nous discuterons simplement deux points : d'abord les relations internes, ensuite la conception du possible comme essentiellement relationnel.

Alix Parmentier (op. cit. p. 309-310) remarque à juste titre que Whitehead admet des relations externes, mais qu'elles doivent être fondées sur l'essence. C'est en ce sens qu'on peut parler d'un essentialisme ${ }^{39}$ de Whitehead. Elle note aussi qu'il existe plusieurs cas où une relation est " interne d'un côté et externe à l'égard de l'autre terme » (p. 310). Elle s'efforce ainsi de justifier ce fait: "si les relations d'une entité actuelle [...] sont toutes internes à cette entité, il ne s'ensuit pas que les relations qu'elle a à une autre entité actuelle soient internes pour cette dernière. Bien au contraire: de deux entités actuelles se succédant dans le temps, la deuxième seule est en relation interne à l'égard de la première » (ibid).

Soit donc deux instants $t_{1}$ et $t_{2}, t_{2}>t_{1}$. $\grave{A} t_{1}, t_{2}$ n'existe pas et donc il ne peut y avoir de relation interne de $t_{1}$ à $t_{2}$. Quand $t_{2}$ existe, il est nécessaire que $t_{1}$ précède $t_{2}$, mais à $t_{1} t_{2}$ n'existant pas ( $t_{2}$ est purement possible), la relation ne peut exister entre $t_{1}$ et $t_{2}$. La raison en est qu'une relation doit porter sur deux choses existantes (principe de symétrie ontologique des relations. ${ }^{40}$ La relation interne est donc orientée [anachroniquement]. Estce que cela veut dire que la relation [diachronique] de $t_{1}$ à $t_{2}$ est externe ? Une manière de comprendre cette asymétrie est de se situer dans un cadre où à $t_{1}$ le temps se ramifie ( $t_{2}$ étant futur) : en effet dans ce modèle $t_{2}$ aurait 
pu appartenir à l'autre branche de la ramification et donc la relation de $t_{2}$ à $\mathrm{t}_{1}$ est externe, car non nécessaire.

Le point fondamental est que si Whitehead n'accepte pas pour les relations internes ce principe de la théorie des relations qui stipule que les propriétés de $\mathrm{R}$ sont héritées par la converse de $\mathrm{R}^{41}$ : dans le cas des relations internes cela donnerait le principe que si $\mathrm{R}$ est interne, la converse de $\mathrm{R}$ est interne, ce qui est précisément ce que Whitehead transgresse. Pour Whitehead toute relation n'a pas de converse. Il y aurait donc une autre manière de comprendre que $\mathrm{R}(\mathrm{A}, \alpha)$ est interne, alors que $\mathrm{R}^{\prime}(\alpha, \mathrm{A})$ est externe : contre toute attente, $\mathrm{R}^{\prime}(\alpha, \mathrm{A})$ ne serait pas la converse de $\mathrm{R}(\mathrm{A}, \alpha)$. Russell et Whitehead dans les Principia Mathematica énoncent cependant ce principe que Whitehead viole en 1926 : «any relation has a converse » (*31. 13, PM, tome 1, p. 238). On pourrait être tenté de concéder au regretté Jules Vuillemin que «sa théorie [celle de Whitehead] contredit la logique, parce qu'elle contredit l'atomisme logique des propositions éléments, dont la valeur de vérité ne dépend que de leur rapport à la réalité, sans être des fonctions de la valeur de vérité de toutes les autres propositions » (1971, p. 96). Toutefois, à un examen plus attentif il ne saurait échapper que l'équivalence posée par J. Vuillemin entre «la logique » et «l'atomisme logique » ne va pas de soi. Le Tractatus ne sert pas à proscrire tout holisme sémantique, mais à tracer les limites de l'atomisme logique et l'on sait qu'il le fait au prix d'un nihilisme sémantique que l'on peut qualifier de «mystique » au sens peu gracieux où Vuillemin l'entend quand il applique cet épithète à Whitehead (op. cit. p. 97).

Je ne ferai qu'esquisser quelques remarques à propos du possible. Pour Whitehead chaque possible est intrinsèquement relationnel. Il ne donne pas cependant d'indications sur les types de relation qui existent entre les possibles. Dans la métaphysique contemporaine c'est la relation d'accessibilité qui structure cette relationalité des mondes et des individus possibles, mais cette relation d'accessibilité suppose que le monde de départ dans la relation ouvre sur une sphère d'accessibilité dans laquelle voisinent des mondes possibles de même rang ou extraction ontologique que le nôtre (même si seul le nôtre est actuel, il a une sorte de point commun avec tous les autres mondes possibles) La conception whiteheadienne $\mathrm{du}$ possible n'est ni combinatoire (Leibniz) ni indexicale (Lewis) ; il considère le possible, comme Aristote, en vue de l'actualisation. En ce sens chaque possible est en relation avec sa réalisation future dans une occasion actuelle. Toutefois quand nous posons cette thèse forte du caractère relationnel du possible nous entendons que chaque possible ait au moins une relation avec un autre possible, que les 
possibles ne soient pas isolés, qu'ils aient une connexion déterminée. Whitehead dans le texte que j'ai commenté ne répond pas à cette question ; il faudra attendre Process and Reality pour qu'il systématise ses vues sur la dynamique de la possibilité et de son actualisation infinie (1929, II, I : Le schème catégorial).

\section{Bibliographie}

Ellis, Brian (2001) : Scientific Essentialism, Cambridge University Press.

Lowe, Victor (1985 \& 1990) : Alfred North Whitehead. The Man and his Work, John Hopkins University Press, Baltimore, 2 vols.

Mondadori, Fabrizio (1973) «Reference, Essentialism and Modality in Leibniz's Metaphysics », Studia Leibnitiana, 5, p. 136-148.

Monnoyer, Jean-Maurice (2003) : « La transparence et le caractère double de l'expérience ", in Philosophies de la perception. Phénoménologie, grammaires et sciences cognitives, Jacques Bouveresse \& Jean-Jacques Rosat éds., Collège de France/ Odile Jacob, p. 173-200.

Nef, Frédéric : (2004) Qu'est-ce que la métaphysique?, Gallimard, Paris.

Nef, Frédéric : (2005a) Les propriétés des choses, Vrin, Paris.

Nef, Frédéric : (2005 b) « Pourquoi quelque chose plutôt que rien? » in Pourquoi existe-t-il quelque chose plutôt que rien?, Francis Wolff éd., ENS/PUF, Paris.

Parmentier, Alix (1968) La philosophie de Whitehead et le problème de Dieu, Beauchesne, Paris.

Peirce, Charles Sanders (1998): The essential Peirce. Selected Philosophical Writings. VoLume I (1893-1913), Peirce Project Edition, éds. Nathan Houser \& Christian Kloesel, Indiana University Press.

Saint-Sernin, Bertrand (2000): Whitehead. Un univers en essai, Vrin, Paris, 2000.

Vernant, Denis (2003) Russell, Flammarion, Paris.

Vuillemin, Jules (1971) : La logique et le monde sensible. Etude sur les théories contemporaines de l'abstraction, Flammarion, Paris [chapitre III : Whitehead].

Whitehead, Alfred North \& Russell, Bertrand (1913 $1^{\text {ère }}$ éd.) Principia Mathematica, t. 1, Cambridge University Press. 
Whitehead, Alfred North (1926) : Science and the Modern World, Lowell Lectures 1925, The MacMillan Company, New York [chapitre 10: Abstraction] (trad. française - peu utilisable - par Paul Couturiau, Editions du Rocher, Monaco, 1994).

Whitehead, Alfred North (1929, éd. corrigée 1978) : Process and Reality. An Essay in Cosmology, éd ; corrigée, éd. David R. Griffin \& Donald W. Sherburne, Gifford lectures 1927-1928, The Free Press, New York (trad. fr. sous la direction de Dominique Janicaud, Gallimard, Paris, 1995).

\section{Notes}

${ }^{1}$ Dans sa critique Jules Vuillemin n'examine en fait que les textes de la première période de la philosophie de Whitehead (surtout Time, Space and Material (1919) et An Inquiry into the Principles of Natural Knowledge (1919).

${ }^{2} \mathrm{Ce}$ chapitre ne concerne pas mon propos qui concerne l'ontologie de la relation entre le général et le particulier et je n'en dirai rien. Pour la théologie de Whitehead voir l'admirable somme de Alix Parmentier (1968) et toute l'œuvre de Charles Hartshorne.

${ }^{3}$ Ceci est confirmé par l'existence d'un exemplaire de Space, Time and Deity copieusement annoté par Whitehead en 1924 (V. Lowe, p. 174). Rappelons que ce chef d'œuvre d'Alexander a été publié en 1920 (cf. Nef 2004, p. 610 ss). Alexander avait lu Bergson (cf. les œuvres de Bergson, Edition du Centenaire, PUF, 1959, p. 15491550), pour une critique de la notion bergsonienne d' « image» dans Matière et Mémoire. J'ai été incapable de déterminer si Bergson a lu Alexander.

${ }^{4}$ On pourrait même risquer l'hypothèse que l'émergence de l'esprit c'est 1 'émergence d'une connaissance de la structure métaphysique, conçue comme comprésence des qualités vitales ou matérielles. La déité en ce sens serait pour Alexander la connaissance de la comprésence des esprits, mais pas conçue au sens d'un corps mystique, mais simplement d'un réseau des relations entre esprits. Pour Whitehead Dieu est au terme du processus d'émergence ; c'est en ce sens qu'il est historique, même s'il est éternel (d'où la compréhension par Whitehead, à la différence de Peirce, McTaggart, Alexander, de la nécessité métaphysique de l'Incarnation, nécessité distincte de sa gratuité dans l'économie du salut). 
${ }^{5}$ Un examen plus approfondi réclamerait une relecture des objections adressées à cette théorie telle qu'elle a été exposée à nouveau par Whitehead quelques années plus tard dans Process and Reality: il faudrait déterminer si dans cet ouvrage il a modifié sa théorie des objets éternels de façon à parer les objections que ne manque pas de faire naître le texte que j' examine ici.

${ }^{6}$ Pour un développement sur la relation entre ingression et participation, voir B. Saint-Sernin (2000), p. 79-87. Il ne semble pas toutefois que l'on puisse suivre cet auteur quand il semble identifier ingression et participation (même s'il est certain que la notion d'ingression a sa source dans Platon). Si $\alpha$ participe à $\mathrm{A}$, alors $\mathrm{A}$ a une relation d'ingression à $\alpha$; si $\mathrm{A}$ a une relation d'ingression à $\alpha$, alors il n'est pas nécessaire que $\alpha$ participe à $\mathrm{A}$. Les déterminés rentrent dans la relation d'ingression, mais la participation se fait au déterminable : cette balle participe au bleu, mais pas à cette nuance de bleu et par contre cette nuance de bleu entre dans une relation d'ingression (cf. infra pour ce point).

${ }^{7}$ Trad. fr. op. cit. p. 144 ; cf. A. Parmentier 1968, p. 69-70.

${ }^{8}$ Il faut distinguer les sense objects des sense data, qui sont des atomes perceptuels.

${ }^{9}$ C f. A. Parmentier, p. 73-79. Cette classification qui dates des années vingt est surtout épistémique. Plus tard Whitehead distinguera dans Process and Reality quatre types d'objets: objets éternels, propositions, entités actuelles objectivées, nexus. (cf. trad. franç. p. 117)

${ }^{10}$ A. Parmentier, p. 298

${ }^{11}$ Un des intérêts de l'étude de Whitehead est de ne pas se laisser enfermer dans le jeu de miroirs de la Forme et de la Substance, opposition toute scolaire et rhétorique, cliché historiographique. cf. les travaux d'A. de Libéra qui prend bien soin de rapporter la querelle des universaux à la tentative de synthèse de Porphyre et à la forme de la question que lui donne cette tentative de synthèse.

${ }^{12}$ On peut considérer Aristote (tout comme Leibniz d'ailleurs) comme un philosophe de l'organisme, qui a raté son objet en confondant les schèmes logiques et les schèmes biologiques.

13 On peut rapprocher ceci de Leibniz, chez qui les possibilités appartiennent au royaume éternel des essences en Dieu. Leibniz pose implicitement une relationalité des essences puisque les 
essences sont des possibles liés au moins par la relation de compossibilité.

${ }^{14}$ C.S. Peirce a posé le problème de la généralité en relation avec le continuum, mais dans un contexte différent, celui de la théorie de l'abduction. Cf. Peirce (1998) p. 238 où il identifie incidemment «true continuity» et «true generality». Cf. sur ce sujet C. Chauviré : Peirce et la logique du vague, PUF, Paris, 1995. Pour la théorie de Whitehead du continuum ; cf Process and Reality, II,2)

${ }^{15}$ La différence entre 'le rouge' et 'la sphéricité' est qu'il existe une nuance de rouge, mais pas de nuance de sphéricité, d'où l'opposition entre l'article indéfini pour la nuance de rouge et l'article défini pour la sphéricité. Cependant même si la sphéricité est une propriété à contours nets (à la différence du rouge), la sphéricité de cette balle est différente de la sphéricité de cette autre balle. Les deux propriétés sont numériquement distinctes, que ses contours soient nets ou pas.

${ }^{16}$ En gros il existe trois approches de l'actualité : (i) existent les choses actuelles, concrètes (cf. le pansomatisme de Kotarbinski), (ii) l'actualité est indexicale, chaque monde possible est actuel pour luimême (réalisme modal de Lewis), (iii) existent les choses actuelles et les objets idéaux. La position (iii) est celle de Whitehead, qui s'oppose à (i) en ce que n'existe pas seulement l'actuel concret et à (ii) en ce que l'actualité est absolue : il y a un seul univers de réalisation.

${ }^{17}$ En ce sens «abstrait» est synonyme de «séparé ». l'intelligible est abstrait, en ceci qu'il est séparé du sensible. Les entités platoniciennes sont abstraites en ce sens.

18 En ce sens «abstrait est synonyme d' «indépendant». Les entités platoniciennes sont abstraites en ce sens également. Cependant pour Whitehead «absolument abstrait» n'a pas de sens et on peut caractériser les Formes de Platon comme absolument abstraites. (cf. note suivante)

19 En cela l'abstraction whiteheadienne est différente de l'aphairasis platonicienne et se rapproche d'Aristote, si on comprend l'inhérence de l'universel au particulier comme une connexion.

${ }^{20}$ La coincidentia oppositorum est appliquée par Nicolas de Cues d'abord et avant tout aux figures mathématiques. Si on fait varier un angle, en augmentant son nombre de degré, viendra un moment où mesurant $180^{\circ}$, il n'y aura plus d'angle, mais une droite et si l'angle 
était celui d'un triangle, on n'aura plus un triangle, c'est à dire une figure fermée composée de droites droites, mais une figure ouverte composée de deux droites. Au moment du changement (le passage de $180^{\circ}-\varepsilon$ où $\varepsilon$ tend vers 0 , à $180^{\circ}$ ) pour Nicolas de Cues il y a coïncidence de l'angle et de la droite, des deux figures. Cf. Maurice Patronier de Gandillac : La philosophie de Nicolas de Cues, Aubier Montaigne, Paris, 1941, p. 208 ss.). Leibniz dans son analyse des fonctions étudiera ce genre de "saut à la limite», mais leur donnera un statut purement «mental» (cf. Leibniz: Naissance du Calcul différentiel, Parmentier éd., Vrin, Paris, 1989, p. 428). Quand il rencontrera une véritable coïncidences des opposés (cabale, yi king), il lui donnera un sens figuré. La coïncidentia oppositorum provient probablement de la mystique rhénane, qui voit par exemple en Dieu un être auprès duquel tout étant est un néant, et un « rien éternel » au-delà de l'essence. De même l'homme est néant, mais par son âme il vaut plus que tout l'univers.

${ }^{21}$ Whitehead voit dans les «objets éternels complexes» des «situations abstraites ». On aurait envie, pour clarifier le développement, de considérer qu'il y a une combinatoire à quatre termes :

objets éternels complexes ou situations abstraites (I) vs. Objets éternels simples (II)

situations concrètes (III) vs. Qualités concrètes exemplifiées dans des occasions actuelles (IV)

Les entités de type I entrent en ingression dans les entités de type II et les entités de type III entrent en ingression dans les entités de type IV. Si cette modélisation était correcte, cela signifierait que l'ontologie de Whitehead repose sur une double opposition: Abstrait vs. Concret et Simple vs. Complexe et qu'un de ses traits importants est de ne pas identifier univoquement et complètement le simple et l'abstrait, le complexe et le concret.

On serait tenté, en usant du lexique métaphysique contemporain d'affirmer que l'objet actuel que je perçois est la combinaison d'un particulier abstrait (l'objet éternel comme abstrait, ayant une relation externe à l'objet actuel) et d'un particulier concret (l'objet comme concrétion de qualités entrant dans une relation d'ingression avec lui). L'objet éternel particularise parce qu'il est individuel, ce n'est pas l'objet actuel qui particularise l'objet éternel, ce n'est pas la substance qui individualise l'accident. 
${ }^{23}$ Cette définition est différente de la définition classique qui fait entrer en jeu la notion de nécessité : $\mathrm{A}$ a une relation interne $\mathrm{R}$ à $\mathrm{B}$, si nécessairement $\mathrm{A}$ a une relation $\mathrm{R}$ à $\mathrm{B}$. on peut se demander si le caractère éternel de la relation interne (cf. immédiatement cidessous) n'est pas équivalent à la nécessité.

${ }^{24}$ Peirce voit les choses à l'inverse, puisqu'il admet du vague réel, au niveau des individus et non des universaux.

${ }^{25} \mathrm{Si}$ nous considérons les conditions énoncées plus haut de la relation interne (détermination, constitutivité, caractère constant), on peut remarquer que la troisième condition semble violée : une occasion actuelle qui est bleue à $t$, peut être verte à $t^{\prime}, t^{\prime}>t$. On y reviendra.

${ }^{26}$ On laisse de côté le fait de savoir si Aristote lui-même n'établissait pas une métarelation beaucoup plus stricte entre les deux. On peut voir par exemple une des sources des difficultés de l'aristotélisme dans la projection de la relation de prédication dans l'exemplification, projection à laquelle échapperait Whitehead par cette thèse de l'asymétrie de l'ingression et de la concrétion (la première interne, la seconde externe).

${ }^{27}$ La notation « Cnv $(\mathrm{R}, \mathrm{S}) »$ est celle des Principia Mathematica. « Cnv $(\mathrm{A}, \mathrm{B}) »$ signifie que A est la converse de B.

${ }^{28}$ Cf. Nef 2005, p. 220.

${ }^{29}$ A supposer qu'il voie dans la perception la préhension synthétique et esthétique de la togetherness Whitehead apporte donc une solution originale au problème complexe de l'objet de la perception : s'il y a une telle préhension de la comprésence des tropes, alors nous percevons leur relation même et la perception a une portée métaphysique immédiate encore plus radicale que dans la phénoménologie. Cf. Nef, 2005a, à paraître (chapitre: "Que percevons-nous?») pour des arguments sceptiques à l'encontre de cette perception directe de la comprésence.

${ }^{30}$ C'est parce que Whitehead se situe dans un cadre actualiste, que cette dernière affirmation peut être soutenue. Dans un cadre possibiliste, dans un monde possible contenant une seule possibilité, cela cesserait d'être vrai. Cela dit, même dans un cadre possibiliste on peut douter de l'existence d'un monde avec une seule possibilité. Si ce monde possible contient un seul état de choses atomique (ce qui est vraiment le minimum - cf. Nef 2005 b, à paraître pour la non existence d'un monde vide) — mettons $\mathrm{Fa}$ - il y a alors au moins 
deux possibilités : $\mathrm{M} \neg \mathrm{Fa}$ et $\neg \mathrm{M} \mathrm{Fa}$; et ces deux possibilités sont liées : $\mathrm{M} \neg \mathrm{Fa} \vee \neg \mathrm{M} F$.

31 On sait que c'est Russell qui a donné une forme canonique à cette difficulté, en y voyant (peut être à tort) un défaut majeur de la théorie idéaliste des relations de Bradley. Cf D. Vernant, Russell, 2003).

32 « Each object stands internally in all its possible relationships » (op. cit.)

${ }^{33} \mathrm{Si}$ on définit l'essentialisme comme la doctrine qui stipule qu'il y a des propriétés essentielles à côté des propriétés accidentelles, le superessentialisme est la doctrine qui stipule que toutes les propriétés sont nécessaires. D'après F. Mondadori (1973) Leibniz a soutenu une forme de superessentialisme (c'est Mondadori, qui d'après B. Mates, a forgé ce terme dans cet article de 1973).

${ }^{34}$ Il faudrait ici comparer la togetherness des objets éternels avec la comprésence des tropes dans les particuliers concrets. En un certain sens les objets éternels sont des particuliers abstraits (au sens défini plus haut de 'abstraits'), mais ils diffèrent des tropes, car ils sont des essences relationnelles, des possibilités en vue d'une actualisation, alors que les tropes, qui sont aussi des particuliers abstraits sont des éléments non nécessairement relationnels et actuels.

${ }^{35}$ Cette épaisseur de la chair, qui la fait préférer à la sécheresse du concept est une illusion qui ne vient que du défaut de connaissance que nous en avons ; elle n'est que pour un entendement qui n'en pénètre pas le tissus relationnel. La connaissance métaphysique, à laquelle il manque une intuition qui lui soit spécifique, est cependant capable de discerner que c'est le caractère limité de la connaissance de l'intuition commune de la comprésence qui en est la racine et le fondement.

${ }^{36}$ J.-M. Monnoyer (2003) a analysé cette notion, que l'on laisse souvent de côté, dans un remarquable article où il débusque toutes les formes d'illusion de la transparence et distingue une transparence phénoménale et une transparence de l'expérience proprement dite.

${ }^{37}$ Le terme «translucency » est choisi de préférence à transparency. La translucency est une transparence relative ; elle ne fait que signaler le passage de la lumière (ce que signifie trans-lucide). La transparency est la neutralité d'un milieu qui ne représente aucun obstacle. 
${ }^{38}$ A ce propos J.-M. Monnoyer montre le caractère double du percevoir par transparence: Perçoit-on à travers la vitre ou perçoit-on la transparence de la vitre ? "Voyons-nous à travers la vitre [...] ou voyons-nous que le verre est transparent?»(op. cit. p. 173). On peut remarquer que nous voyons les deux.

${ }^{39}$ Le terme 'essentialisme' n'a, ici au moins, rien de péjoratif. Quine a donné à ce terme son sens trivial, en désignant par son usage la confusion conceptuelle consistant à postuler une distinction (accidentel/essentiel) sans posséder de critère pour valider ou invalider cette distinction dans des cas empiriquement testables. Cette critique quinienne est largement solidaire d'un état de la métaphysique et de la logique philosophique où effectivement un tel critère ne pouvait être donné, ce qui était en fait, une condamnation beaucoup plus du cadre théorique dans lequel on tenait à se maintenir, qu'à la distinction elle-même et à son caractère malsain. B. Ellis (2001) a fourni récemment une brillante défense de la compatibilité de l'essentialisme avec la science moderne et les conditions de rigueur auxquelles elle soumet la métaphysique.

${ }^{40}$ Pour une discussion de ce principe, cf. Nef 2005a, à paraître.

${ }^{41}$ Par exemple si «fils de » est non symétrique et si «père de » est la converse de « fils de », alors « père de » est non symétrique. 
